\title{
LINEAR OPERATORS WHOSE DOMAIN IS LOCALLY CONVEX
}

\author{
by N. J. KALTON \\ (Received 6th October 1975)
}

\section{Introduction}

Let $F$ be an arbitrary topological vector space; we shall say that a subset $S$ of $F$ is quasi-convex if the set of continuous affine functionals on $S$ separates the points of $S$. If $X$ is a Banach space and $T: X \rightarrow F$ is a continuous linear operator, then $T$ is quasi-convex if $\overline{T(U)}$ is quasiconvex, where $U$ is the unit ball of $X$.

In the case when $T$ is compact, $\overline{T(U)}$ is quasi-convex if and only if it affinely homeomorphic to a subset of a locally convex space. This is immediate, since the topology on $\overline{T(U)}$ can be induced by the family of affine functionals vanishing at $\mathbf{0}$. It is also equivalent to the condition that 0 has a base of convex neighbourhoods in $\overline{T(U)}$; this is proved by constructing on the linear span of $\overline{T(U)}$ the finest vector topology $\gamma$ agreeing with the given topology on $\overline{T(U)}$. Then $\gamma$ is locally convex-this follows from results in $(21$, p. 51$)$.

In (16) Peck and Waelbroeck ask whether every compact convex set is locally convex. An equivalent question is whether every compact operator is quasi-convex. This question has recently been resolved negatively by $J$. W. Roberts (unpublished). We obtain some partial results here (for results on this problem in a different direction, see (9)). If $X$ is reflexive, every bounded operator is quasi-convex, while if $X^{*}$ has the Radon-Nikodym property, every compact operator is quasi-convex. Thus if $K$ is a compact convex set such that the Banach space $A_{b}(K)$ of all bounded affine functions on $K$ has the Radon-Nikodym property, $K$ is strongly locally convex (in the terminology of (9)), i.e. affinely homeomorphic to a subset of a locally convex space.

We are also able to relate quasi-convexity of the range of a vector measure to the existence of a control measure. In 1947, Maharam (13) asked whether a sequentially continuous submeasure on a $\sigma$-algebra is equivalent to a measure. Applying the above results we relate Maharam's problem to the question of quasi-convexity of exhaustive operators on spaces $C(\Omega)$ (see $(11)$ ).

The author would like to thank J. Diestel, L. Drewnowski and Z. Lipecki for several helpful comments on a preliminary version of this note. 


\section{Quasi-convex sets and operators}

Suppose $E$ is an $F$-space (complete metric linear space) with the metric topology denoted by $\tau$. Then a vector topology $\rho$ on $E$ such that $\rho \leqslant \tau$ is compatible (with $\tau$ ) if $\tau$ and $\rho$ define the same closed subspaces (10). If $\rho$ is compatible then $\tau$ is $\rho$-polar (i.e. has a base of $\rho$-closed neighbourhoods of 0) by (10) Corollary 5.3.

Lemma 2.1. Let $E$ and $F$ be F-spaces and $T: E \rightarrow F$ be a continuous linear operator. Let $\rho$ be a compatible topology on $E$ and suppose $\left(x_{n}\right)$ is a sequence in $E$ such that $x_{n} \rightarrow 0(\rho)$. If $\left(T x_{n}\right)$ converges then $T x_{n} \rightarrow 0$.

Proof. Let $\|\cdot\|$ denote $F$-norms on either $E$ or $F$ defining the metric topologies. If $x_{n} \rightarrow 0(\tau)$, then $T x_{n} \rightarrow 0$. Thus we may assume $\left\|x_{n}\right\| \geqslant \epsilon>0$ for all $n$. Then by Theorem 3.1 of (10), we may determine a subsequence $\left(z_{n}\right)$ of $\left(x_{n}\right)$ such that $\left(z_{n}\right)$ is basic in $E$, and

$$
\left\|T z_{n}-\omega\right\| \leqslant 2^{-n}
$$

where $\omega=\lim _{n \rightarrow \infty} T z_{n}$. Let $u_{n}=T z_{n}-\omega$, and suppose $\omega \neq 0$.

Suppose $\sum c_{n} z_{n}$ converges; then as $\left\|z_{n}\right\| \geqslant \epsilon>0, \lim _{n \rightarrow \infty} c_{n}=0$. Thus $\Sigma c_{n} u_{n}$ converges, and hence so does $\Sigma c_{n} \omega=\Sigma c_{n} T x_{n}-\Sigma c_{n} u_{n}$; this means that $\Sigma c_{n}$ converges. We may define a linear functional $\psi$ on the closed linear span of $\left(z_{n}: n \in \mathbb{N}\right)$ by

$$
\psi\left(\sum_{n=1}^{\infty} c_{n} z_{n}\right)=\sum_{n=1}^{\infty} c_{n}
$$

and $\psi$ is continuous by the Banach-Steinhaus Theorem for $\tau$. As $\rho$ is compatible, $\psi$ is $\rho$-continuous; since $\psi\left(z_{n}\right)=1$ and $z_{n} \rightarrow 0(\rho)$ we have a contradiction.

Theorem 2.2. Let $E$ and $F$ be F-spaces and $T: E \rightarrow F$ be a continuous linear operator. Let $\rho$ be a compatible topology on $E$ and $S \subset E$ a $\rho$ compact set. Then

(i) $T(S)$ is closed in $F$

(ii) If $T$ is injective, $T^{-1}$ is continuous on $T(S)$ for the topology $\rho$ on $E$.

Proof. (i) Suppose $\omega \in \overline{T(S)}$ and $x_{n} \in S$ are such that $T x_{n} \rightarrow \omega$. Then, as $S$ is $\rho$-sequentially compact (see (10)), there is subsequence $\left(z_{n}\right)$ of $\left(x_{n}\right)$ such that $z_{n} \rightarrow z \in S$. Then $T\left(z_{n}-z\right) \rightarrow \omega-T z$ and so by Lemma $2.1 \omega=$ $T z \in T(S)$.

(ii) If $T x_{n} \rightarrow T x$ and $x_{n} \rightarrow x,(\rho)$, then we again appeal to the $\rho$-sequential compactness of $S$ to deduce that there is a subsequence $\left(z_{n}\right)$ of $\left(x_{n}\right)$ such that $z_{n} \rightarrow z \neq x$. It follows from Lemma 2.1 that $T x=T z$, a contradiction. 
We now restrict our attention to the case when $E$ is locally convex, and $\rho$ is the weak topology on $E$. The following theorem extends Proposition 5.11 of (3).

Theorem 2.3. Let $E$ be a locally convex space and $F$ a topological vector space. Suppose $T: E \rightarrow F$ is continuous. If $S$ is a weakly compact subset of $E$, then $T(S)$ is closed and quasi-convex.

Proof. First suppose $F$ is an $F$-space. Then we factorize $T$ thus:

$$
E \stackrel{A}{\rightarrow} M \stackrel{B}{\rightarrow} F
$$

where $M$ is metrizable and locally convex and $B$ is injective (it is easy to see that this is possible). Then $A(S)$ is weakly compact in $M$ and hence $T(S)$ is closed. If $\phi \in M^{*}$ then $\phi \circ B^{-1}$ is continuous on $T(S)$ by Theorem 2.2 , and the set of such affine functionals separate the points of $T(S)$.

The case of general $F$ follows by embedding in a product of $F$-spaces.

\section{Operators on Banach spaces}

Now suppose $X$ is a Banach space. Theorem 2.3 yields:

Proposition 3.1. Every continuous operator on a reflexive Banach space is quasi-convex.

It is natural, as noted in the introduction, to ask about compact operators. Here we can extend the class of Banach spaces somewhat.

Theorem 3.2. Suppose $X$ is a Banach space such that $X^{*}$ has the Radon-Nikodym property. Then every compact operator on $X$ is quasiconvex.

Proof. Suppose $T: X \rightarrow F$ is a compact operator. It is enough to consider the case when $F$ is an $F$-space. Let $U$ denote the unit ball of $X$. If $T$ fails to be quasi-convex, then 0 does not possess a base of convex neighbourhoods in $\overline{T(U)}$. Thus there exists $\epsilon>0$ such that $\{x:\|x\|<\epsilon\}$ contains no convex neighbourhood in $\overline{T(U)}$. We may, therefore, find $\omega_{n} \in \overline{T(U)}$ such that $\omega_{n} \rightarrow 0$, but $\overline{c o}\left\{\omega_{n}, \omega_{n+1}, \ldots\right\} \cap\{x:\|x\| \geqslant \epsilon\}$ is non-empty for each $n$. Choose $u_{n} \in U$ such that $\left\|T u_{n}-\omega_{n}\right\| \leqslant \epsilon \cdot 2^{-(n+2)}$; then $\overline{c o}\left\{T u_{n}\right.$, $\left.T u_{n+1}, \ldots\right\} \cap\left\{x:\|x\| \geqslant \frac{1}{2} \epsilon\right\}$ is non-empty for each $n$. If $X_{0}$ is the closed linear span of $\left\{u_{1}, u_{2}, u_{3}, \ldots\right\}$, then the restriction of $T$ to $X_{0}$ also fails to be quasi-convex.

Thus we may suppose $X$ separable, and in this case $X^{*}$ is also separable (Stegall (18)). Suppose $x^{* *} \in X^{* *}$; then there is a sequence $x_{n} \in X$ such that $x_{n} \rightarrow x^{* *}$ in the weak*-topology of $X^{* *}$. For any pair of increasing sequences of integers $\left(p_{n}\right)$ and $\left(q_{n}\right) x_{p_{n}}-x_{q_{n}} \rightarrow 0$ weakly and hence (Lemma 2.1) $T\left(x_{p_{n}}-x_{u_{n}}\right) \rightarrow 0$. Thus $\left(T x_{n}\right)$ is a Cauchy sequence; 
define $\tilde{T} x^{* *}=\lim _{n \rightarrow \infty} T x_{n}$. It is easy to see that this definition does not depend on the choice of $\left(x_{n}\right)$, by interlacing sequences. Thus we define a linear map $\tilde{T}: X^{* *} \rightarrow F$. We claim that $\tilde{T}$ is continuous for the bounded weak*topology on $X^{* *}$ (i.e. the finest vector topology agreeing with the weak*topology on the unit ball $U^{* *}$ of $X^{* *}$ ). It is enough to show that $\bar{T}$ is weak*-continuous on $U^{* *}$, and $U^{* *}$ is weak*-metrizable. Suppose $u_{n}^{* *} \in$ $U^{* *}$ and $u_{n}^{* *} \rightarrow 0$ weak*; then select $u_{n} \in U$ such that $u_{n}^{* *}-u_{n} \rightarrow 0$ and $\tilde{T} u_{n}^{* *}-T u_{n} \rightarrow 0$. Then $u_{n} \rightarrow 0$ weakly and hence $T u_{n} \rightarrow 0$, and $\tilde{T} u_{n}^{* *} \rightarrow 0$.

Since $X^{* *}$ with the bounded weak*-topology is locally convex with dual $X^{*}$, the result now follows from Theorem 2.3 , since $\tilde{T}(U)=\overline{T(U)}$.

Remark. Of course the hypotheses of the Theorem fail for $l_{1}$, and the Theorem fails in this case by the example of Roberts.

Corollary 3.3. Let $K$ be a relatively compact convex set in a topological vector space, such that $A_{b}(K)$ has the Radon-Nikodym property. Then $K$ is locally convex.

Proof. We may suppose $0 \notin K$; let $U=\operatorname{co}(K \cup-K)$. Let $X$ be the linear span of $K$ normed by the Minkowski functional of $U$. Then $X^{*} \cong$ $A_{b}(K)$ and the result follows.

Remark. As $A_{b}(K)$ is a dual space, it is sufficient for $A_{b}(K)$ to be weakly compactly generated (see (17)).

\section{Applications to vector measures}

Let $\mathscr{S}$ be a $\sigma$-algebra of sets. Then a submeasure $\nu$ on $\mathscr{S}$ is a map: $\nu: \mathscr{S} \rightarrow \mathbb{R}$ such that $\nu(P) \geqslant 0, P \in \mathscr{S}$ and $\nu(P) \leqslant \nu(P \cup Q) \leqslant \nu(P)+\nu(Q), P$, $Q \in \mathscr{S}$. We say $\nu$ is order-continuous if $P_{n} \downarrow 0$ implies $\nu\left(P_{n}\right) \downarrow 0$. Maharam (13) has asked whether every order-continuous submeasure $\nu$ is equivalent to a finite positive measure $\lambda$, i.e. does there exist $\lambda$ such that $\nu\left(P_{n}\right) \rightarrow 0$ if and only if $\lambda\left(P_{n}\right) \rightarrow 0$ ? (see (7) and (13)).

If $F$ is an $F$-space and $\mu: \mathscr{S} \rightarrow F$ is a vector measure, then the semi-variation $\|\mu\|$ defined by

$$
\|\mu\|(P)=\sup (\|\mu(Q)\|: Q \subset P)
$$

is an order-continuous submeasure. A finite positive measure $\lambda$ dominates $\mu$ if $\lambda\left(P_{n}\right) \rightarrow 0$ implies $\|\mu\|\left(P_{n}\right) \rightarrow 0$ and is a control measure for $\mu$ if $\lambda$ is equivalent to $\|\mu\|$ (see (4), (7)). A classical theorem of Bartle, Dunford and Schwartz (2) states that if $F$ is a Banach space then $\mu$ has a control measure. This has been extended by various authors to locally convex settings ((7), (8), (12), (14); see also (15)). The validity of the BartleDunford-Schwartz theorem in general $F$-spaces is equivalent to Maharam's 
problem. One direction of this statement is obvious; for the other observe that if $\nu$ is an order-continuous submeasure, then the space $M(S)$ of bounded $\mathscr{S}$-measurable functions with the topology of convergence in $\nu$-measure is a metric linear space. The map $\mu(P)=\chi_{P}$ (characteristic function of $P$ ) is a vector measure whose semivariation is equivalent to $\nu$.

In this context, we show that local convexity is necessarily involved in the existence of control measures. We assume here that our measure $\mu$ has the property that the convex hull of its range is bounded. For this property, Turpin (20) uses the term $L_{\infty}$-bounded.

Theorem 4.1. Let $F$ be an F-space and $\mu: \mathscr{S} \rightarrow F$ be an $L_{\infty}$-bounded vector measure. Suppose $\lambda$ is a control measure for $\mu$. Then

$$
\overline{c o}\{\mu(P): P \in \mathscr{P}\} \text { is quasi-convex. }
$$

Proof. Consider the space $L_{\infty}(\lambda)$ of $\mathscr{S}$-measurable, $\lambda$-essentially bounded functions. The $L_{\infty}$-boundedness of $\mu$ implies that for $\phi \in L_{\infty}(\lambda)$ we can define $\int \phi d \mu$ (see Turpin (20) Ch. VII). Consider the map $J: L_{\infty}(\lambda) \rightarrow F$ defined by

$$
J(\phi)=\int \phi d \mu .
$$

By the theorem of dominated convergence (Turpin (20) 7.3.7), if $\phi_{n} \rightarrow \phi$ in $\lambda$-measure and sup $\left\|\phi_{n}\right\|<\infty$, then $J\left(\phi_{n}\right) \rightarrow J(\phi)$. Let $\gamma$ be the finest vector topology on $L_{\infty}(\lambda)$ which agrees with the topology of convergence in $\lambda$-measure on bounded sets. Then $\gamma$ is a two-norm topology, since if $\left\|\phi_{n}\right\| \leqslant K$ and $\phi_{n} \rightarrow \phi$ in $\lambda$-measure then

$$
\int\left|\phi-\phi_{n}\right| d \lambda \rightarrow 0
$$

by the bounded convergence theorem. Thus $\gamma$ is induced on the unit ball by the $L_{1}$-norm. It follows that $\gamma$ is locally convex (see (21)) and from the result of (1), 4.2, on the dual of two-norm spaces, $\left(L_{\infty}(\lambda), \gamma\right)^{\prime}=L_{1}(\lambda)$. In fact $\gamma$ is the Mackey topology $\tau\left(L_{\infty}(\lambda), L_{1}(\lambda)\right)$-see (6) or (19). By Theorem 2.3, it follows that $\overline{c o}\{\mu(P): P \in \mathscr{S}\}$ is quasi-convex.

We conclude by observing the relationship with exhaustive operators on spaces $C(\Omega)(11)$. If $\Omega$ is a compact Hausdorff space and $T: C(\Omega) \rightarrow F$ is exhaustive, then $T$ can be expressed as

$$
T f=\int_{\Omega} f d \mu
$$

where $\mu$ is an $L^{\infty}$-bounded $F$-valued measure on the Borel sets of $\Omega$. Quasiconvexity of $T$ is then equivalent to the existence of a control measure for $\mu$. Conversely if $\nu$ is a order-continuous submeasure on the Borel sets of $\Omega$ then consider the natural inclusion $J: C(\Omega) \rightarrow M_{\nu}(\Omega)$, where 
$M_{\nu}(\Omega)$ is the space of a bounded Borel functions on $\Omega$ with the topology of convergence in $\nu$-measure. $J$ is exhaustive, and the quasi-convexity of $J$ is equivalent to the existence of a control measure for $\nu$.

\section{REFERENCES}

(1) A. Alexiewicz and Z. SEmadeni, Linear functionals on two-norm spaces, Studia Math. 17 (1958), 121-140.

(2) R. G. BARTLE, N. DUNFORd and J. T. SCHWARTZ, Weak compactness and vector measures, Canad. J. Math. 7 (1955), 289-305.

(3) C. Bessaga and A. PElCZyński, The estimated extension theorem, homogeneous collections and skeletons, and their applications to the topological classification of linear metric spaces and convex sets, Fund. Math. 59 (1970), 113-190.

(4) J. K. BROOKS, On the existence of a control measure for strongly bounded vector measures, Bull. Amer. Math. Soc. 77 (1971), 999-1001.

(5) J. P. R. Christensen and W. HERER, On the existence of pathological submeasures and the construction of exotic topological groups, Math. Ann. 213 (1975), 203-210.

(6) J. DAZORD and M. Jourlin, U ne topologie mixte sur l'espace $L_{\infty}, P u b l$. Dept. Math. Lyons 11, 2 (1974), 1-18.

(7) L. DREWNOWSKI, On control submeasures and measures, Studia Math. 50 (1974), 203-224.

(8) J. Hoffman-Jorgensen, Vector measures, Math. Scand. 28 (1971), 5-32.

(9) R. E. JAMISON, R. C. O'BRIEN and P. D. TAYLOR, On embedding a compact convex set into a locally convex topological vector space, Pacific J. Math. (to appear).

(10) N. J. KALtON, Basic sequences in F-spaces and their applications, Proc. Edinburgh Math. Soc. 19 (1974), 151-167.

(11) N. J. KALton, Exhaustive operators and vector measures, Proc. Edinburgh Math. Soc. 19 (1975), 291-300.

(12) I. LabUdA, Sur la theoreme de Bartle-Dunford-Schwartz, Bull. Acad. Polon. Sci. 20 (1972), 557-561.

(13) D. MAHARAM, An algebraic characterization of measure algebras, Ann. of Math. 48 (1947), 154-167.

(14) K. MUsial, Absolute continuity of vector measures, Coll. Math. 27 (1973), 319-321.

(15) K. MUSIAL, Absolute continuity and the range of group-valued measures, Bull. Acad. Polon. Sci. 21 (1972), 105-113.

(16) N. T. PeCK and L. WAelbroeck, Problem 58, Proceedings of the International Colloquium on Nuclear Spaces and Ideals in Operator Algebras, Warsaw, 18-25 June 1969, Studia Math 48 (1970), 482. 
(17) R. R. PHELPS, Dentability and extreme points in Banach spaces, $J$. Functional Analysis 17 (1974), 78-90.

(18) C. Stegall, The Radon-Nikodym property in conjugate Banach spaces, Trans. Amer. Math. Soc. 206 (1975), 213-223.

(19) K. D. STROY AN, A characterization of the Mackey uniformity on $\left(L_{\infty}, L_{1}\right)$ for finite measures, Pacific J. Math. 49 (1973), 223-228.

(20) P. TURPIN, Convexites dans les espaces vectorials topologiques generaux (These, Orsay, 1974).

(21) A. WIWEgER, Linear spaces with mixed topology, Studia Math. 20 (1961), 47-68.

University College of Swansea,

Singleton PARK,

SWANSEA. SA2 8PP 